\title{
The VIMOS VLT Deep Survey: A census of active and passive galaxies to redshift $\mathbf{1 . 3}$
}

\author{
D. Vergani ${ }^{1}$. On behalf of the VVDS collaboration \\ ${ }^{1}$ INAF-IASF Milan, Via E. Bassini 15, I-20100 Milano (Italy) \\ email: daniela@lambrate.inaf.it
}

\begin{abstract}
We are studying how stellar masses assemble through cosmic time since the Universe had only $30 \%$ of its present age. We are conducting a census of galaxies which covers the end of the most active star-forming phase, using a mass-limited sample of approximately 5,000 objects selected from the VIMOS VLT Deep Survey (VVDS) in the redshift range $0.45<z<1.3$. With a criterion based on the direct spectral measurement of the $4000 \AA$ Balmer break, $D_{n}(4000)$, we have classified our sample in spectroscopically early and late-type galaxies. The trends existing between stellar mass, spectroscopic classification, and star formation activity are clearly shown in our analysis.
\end{abstract}

Keywords. cosmology: observations, galaxies: evolution.

\section{Summary}

Understanding the rôle played by the stellar mass in regulating the active phase of starformation is one of the key problems in modern cosmology. In the past, estimates of the star-formation have been deduced using a selection of galaxies with different limitations. Therefore, investigating the location and properties of mass assembly in a statistically meaningful sample of a spectroscopic survey and using as a proxy of the star-formation activity the direct measurement of the equivalent width of the [OII] $\lambda 3727$ emission line contains the key to deduce unambiguously the galaxy evolution.

Using composite stellar population synthesis models and a set of delayed exponential star formation histories $\left(\mathrm{e}^{-t / \tau}\right)$, we found that galaxies with values of the $4000 \AA$ Balmer break lower than $\mathrm{D}_{n} 4000 \leqslant 1.5$ are characterized by an increasing star formation activity with time $(\tau>3.6)$ and defined as spectroscopic late type (SLT) galaxies. We assign a spectroscopic early type (SET) classification to a galaxy with $\mathrm{D}_{n} 4000$ larger than 1.5 and with a quasi-instantaneous burst $(\tau<3.6)$.

Extending similar results obtained in the local Universe (Kauffmann et al. 2003, MNRAS 341, 54), we observed the predominance of SLT galaxies for low-mass systems and a SET classification for the most massive systems up to $z=1.3$. While the low-mass $\left(<10^{10} M_{\odot}\right)$ SET galaxies do not significantly evolve and represent a minority $(<10 \%)$ in SET class, the most massive SET galaxies reveal an evolutionary trend.

SLT galaxies with different stellar mass content share the same decline in the star formation activity as globally presented in several studies (e.g. Lilly et al. 1996, ApJ 460, L1; Madau et al. 1996, MNRAS 283, 1388). An anti-correlation between the strength in the star formation activity and the stellar content is observed in SLT galaxies. The mass proportionally decreases at the increase of star formation activity measured with the mean of equivalent width for the $[\mathrm{OII}] \lambda 3727$ line. The only exception is represented by the most massive objects at redshifts $z<1$; their formation of stars is nearly absent below $z=1$ when their quiescent state changes radically and their contribution to the mass assembly becomes comparable to the less massive systems. 